\title{
Some Aspects of the Search for Invariants
}

\author{
By Dr. G. W. SCOTT BLATR
}

ABSTRACT' of Paper read on 14th November, 1949

Craig defines "invariance" as " the constancy of some complex entity or object of thought while certain of its constituent elements or associated quantities undergo change ". Only the simplest physical properties, such as density, owe their invariance entirely to homogeneity. Thus, for example, the invariance of an elastic modulus depends on a convenient interaction of repulsive and attractive atomic forces. Such physical properties are defined by finding experimentally what powers of (normally) mass, length and time will give an invariant grouping, and so establishing a physical law. The same groupings are not invariant for all types of materials ( $c f$. viscosity and rigidity modulus, conductance and dielectric constant).

Many physical properties only remain invariant for limited changes in their defining elements. The viscosity of the so-called true fluids is probably not constant for very high rates of shear nor does any real metal show ideally elastic behaviour. Some liquids have an invariant viscosity as measured by a falling sphere, but a variable viscosity when flowing through a tube.

Such facts have led some physicists to reject the "physical property" as a useful concept, except for empirical purposes, but even the alternative operational definitions are not devoid of difficulty.

Industrial physicists are often obliged to look for other types of invariance or partial invariance and, to this end, three lines of approach are discussed.

The classical analytical approach explains complex phenomena by integrating or summing the behaviour of postulated idealized components of the system. There is a recent tendency to introduce complex components to give a partial simplification, but this should only serve as an interim solution.

The second method is concerned with those many empirical industrial tests which do not measure invariant properties but rather replicable modes of behaviour under arbitrarily selected processes. Factor Analysis, however imperfect as a statistical method, has certainly thrown useful light on similar problems in Psychometry, and preliminary applications to physical tests are most encouraging. The usefulness of the method depends on the fact that a matrix of correlation coefficients between empirical tests and/or subjective quality scores will usually show some limited regularities. The correlation matrix may be "reduced" by replacing the unities in the diagonal cells by communalities and can then be expressed as the product of a " common factor matrix" and its transpose. The divergence of the common factor matrix from squareness is a measure of the saving effected by the treatment. The analysis may be applied either to relationships between tests (R-technique) or between samples ( $Q$-technique).

Alternatively, each test (R-technique) may be pictured as a vector in multidimensional space, the correlation coefficients between each pair of vectors being given by their scalar products. A set of (normally) orthogonal axes represents the common factors. Although such factors are not entirely invariant to changes in the composition of test-batteries, and perhaps even of sample population, they have a measure of stability which often renders them useful as principles of classification.

The third approach depends on the concept of "quasi-properties". The groupings of mass, length and time found to be invariant for complex systems will differ for different groups of materials, and will normally be found intermediate between those of the simple prototype systems. The quasi-property includes as an inseparable unit ("Gestalt ") not only an intensity, but also one 
or more quality-exponents which indicate the divergence from prototype behaviour. Often a wide group of materials shows an invariant having the same quality-exponents within the limits of error, in which case the quasiproperty hardly differs from a classical physical property. Illustrations are taken from rheology and from physical chemistry.

The method of factor analysis and the use of quasi-properties are compared and shown to have much in common, but it is concluded that only in trivial cases will they lead to the same type of quasi-invariant. 